Supporting Information

\title{
Bi-directional Core Sandwich Structure of Reduced Graphene Oxide and Spinnable Multiwalled Carbon Nanotubes for Electromagnetic Interference Shielding Effectiveness
}

Duck Weon Lee ${ }^{1,2 \ddagger}$, Hyunsoo Kim ${ }^{1 \ddagger}$, Jae Sang Hyeon ${ }^{1}$, Ji Hwan Moon ${ }^{1}$, Bum-Joon Kim ${ }^{1}$, JaeHun Jeong ${ }^{1}$, Junggi Choi ${ }^{1}$, Ray H. Baughman ${ }^{3}$, Geoffrey M. Spinks ${ }^{4}$, Gordon G. Wallace ${ }^{4}$, and Seon Jeong $\mathrm{Kim}^{1 *}$

${ }^{1}$ Center for Self-Powered Actuation, Department of Biomedical Engineering, Hanyang University, Seoul 04763, Korea

${ }^{2}$ Department of Chemistry and Material Science, Aalto University, PO Box 16100, FI-00076 AALTO, Finland

${ }^{3}$ The Alan G. Mac Diarmid NanoTech Institute, University of Texas at Dallas, Richardson, TX 75083, USA.

${ }^{4}$ Intelligent Polymer Research Institute, ARC Centre of Excellence for Electromaterials Science, AIIM Facility, Innovation Campus, University of Wollongong, North Wollongong, NSW 2522, Australia

$\$$ D. W. Lee and H. Kim contributed equally to this work

*To whom correspondence should be addressed, E-mail: sjk@hanyang.ac.kr 
a)

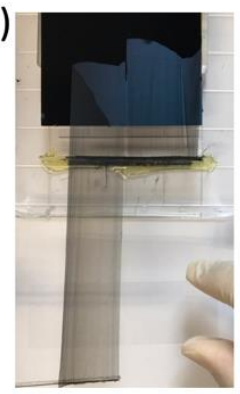

b)

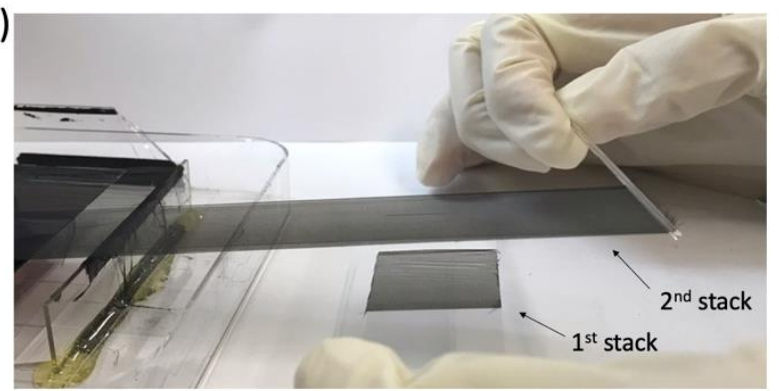

c)

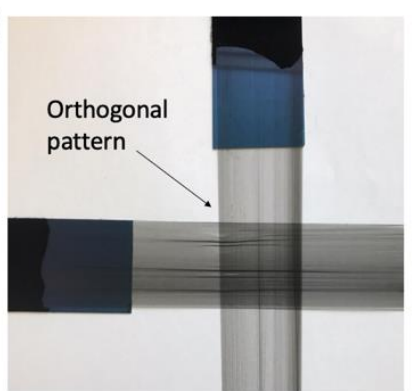

Figure S1. a) The top view, b) side view of the MWNT sheet from the spinnable MWNT forest, and c) top view of the orthogonal pattern of the MWNT sheets from the spinnable MWNT forest. 
EMI SE was measured with a Keysight X11644A calibration kit (Keysight Co. Ltd.) and an 8720D vector network analyzer (Hewlett Packard Co. Ltd.). The EMI SEs of AMSGO and its relevant materials were carried out in a WR-90 rectangular waveguide using a two port network analyzer in the X-band frequency range (8.2-12.4 GHz) as shown in Figure S2. The sample holder was tightly fixed between the both adaptors with four screws and two controllers. The length of the sample holder was $9.78 \mathrm{~mm}$, and the distance from sample to port 1 was set as 0 . The electromagnetic waves had an incident power of $0 \mathrm{dBm}$, which corresponds to $1 \mathrm{~mW}$. Scotch tape was used to insert the sample into the sample holder and was attached to one end of the film to strongly hold it in place. Best care was taken to not alloy any leakage from each edge between ASMGO and the sample holder (the EMI SE of the Scotch tape was ' 0 ').

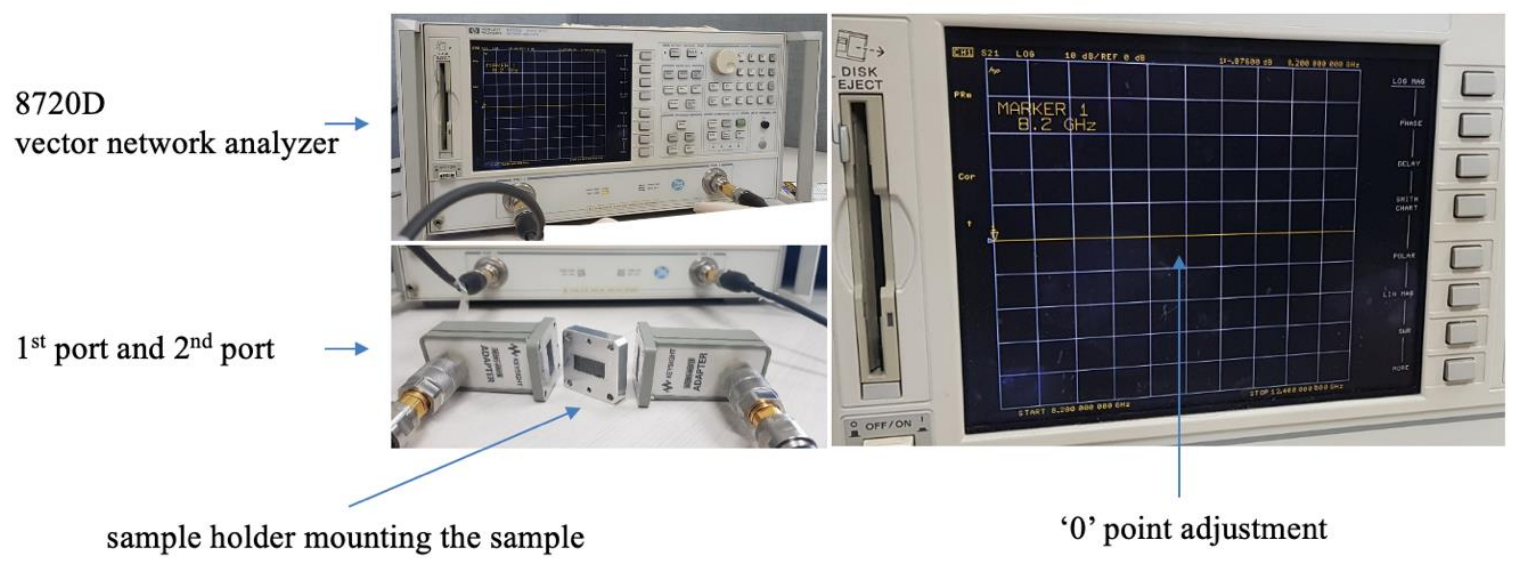

Figure S2. Optical images of a) the $8720 \mathrm{D}$ vector network analyzer (VNA) with $1^{\text {st }}$ port and $2^{\text {nd }}$ port, and a sample holder with the sample mounted, b) " 0 " point adjustment adapters for the $1^{\text {st }}$ port and $2^{\text {nd }}$ port of the $8720 \mathrm{D}$ VNA. 
EMI SE was defined as the ratio of incident to transmitted energy and then expressed in logarithmic scale according to the following Equation (1);

EMI SE $\mathrm{E}_{\text {total }}=10 \log \left(\mathrm{P}_{\mathrm{t}} / \mathrm{P}_{\mathrm{i}}\right)=20 \log \left(\mathrm{E}_{\mathrm{t}} / \mathrm{E}_{\mathrm{i}}\right)$

$$
=\mathrm{SE}_{\mathrm{R}}(\text { Reflection })+\mathrm{SE}_{\mathrm{A}}(\text { Absorption })+\mathrm{SE}_{\mathrm{M}} \text { (Multiple Reflection) }(\mathrm{dB})
$$

where $\mathrm{P}_{\mathrm{i}}\left(\mathrm{E}_{\mathrm{i}}\right)$ and $\mathrm{P}_{t}\left(\mathrm{E}_{\mathrm{t}}\right)$ are the power (electric field) of incident and transmitted EM radiation. Generally, $\mathrm{SE}_{\mathrm{M}}$ is caused by a scattering effect; however, it could be ignored when the thickness of the sample was greater than that of the skin depth $(\delta)$. Equation (2) defines the relationship among transmittance $(\mathrm{T})$, reflectance $(\mathrm{R})$, and absorbance $(\mathrm{A})$;

$\mathrm{T}+\mathrm{R}+\mathrm{A}=1$

In Equation (2), transmittance can be explained by incident and transmitted electric fields;

$\mathrm{T}=\frac{E_{t}^{2}}{E_{i}^{2}}=\mid\left. S_{21}\left(\right.$ or $\left.S_{12}\right)\right|^{2}$

where, $S_{21}$ (or $S_{12}$ ) is a scattering parameter (S-parameter) of transmittance or reflection. Therefore, the relationship between the EMI SE of ASMGO and transmittance is represented in the following graph;

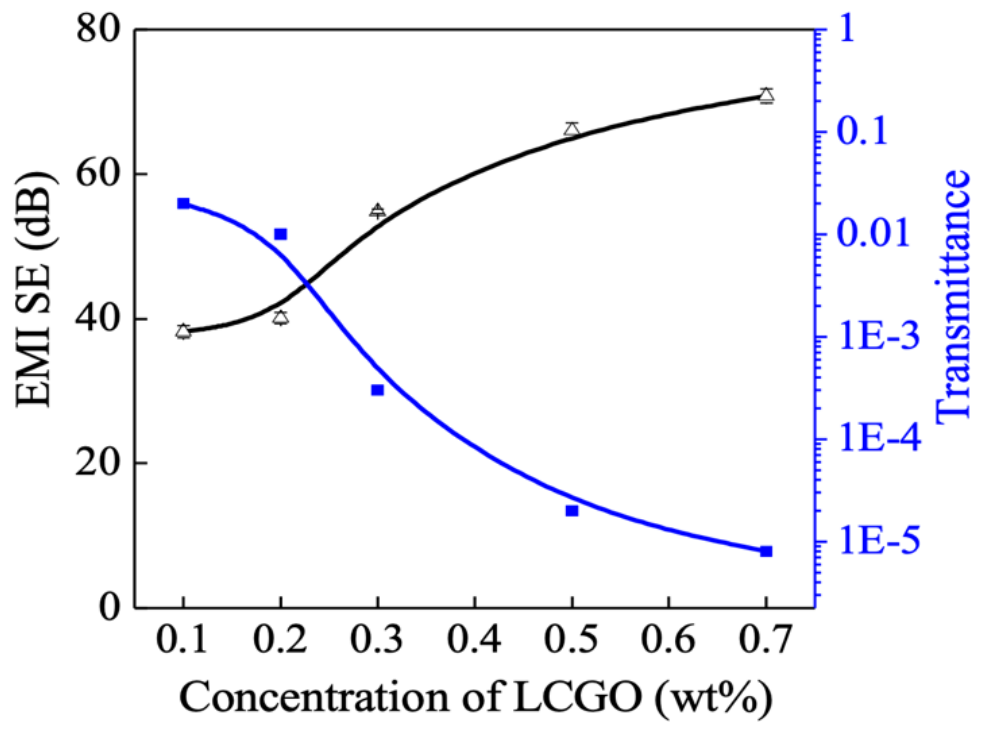

Figure S3. Relationships between the EMI SE in accordance with concentration of r-LCOG and transmittance regarding its power source. 


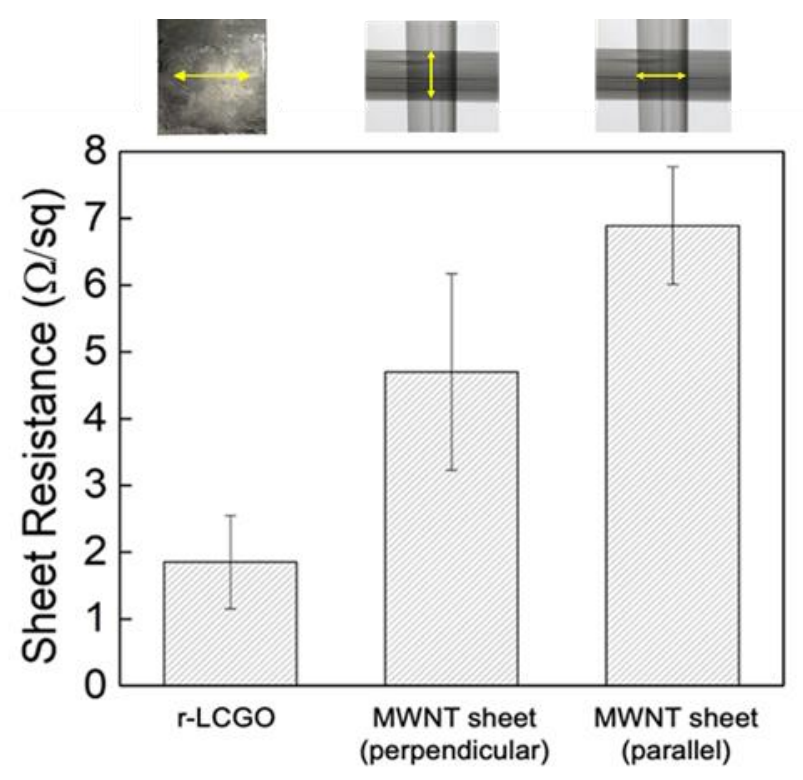

Figure S4. The sheet resistance for r-LCGO and S-MWNTs constituting of ASMOG. 


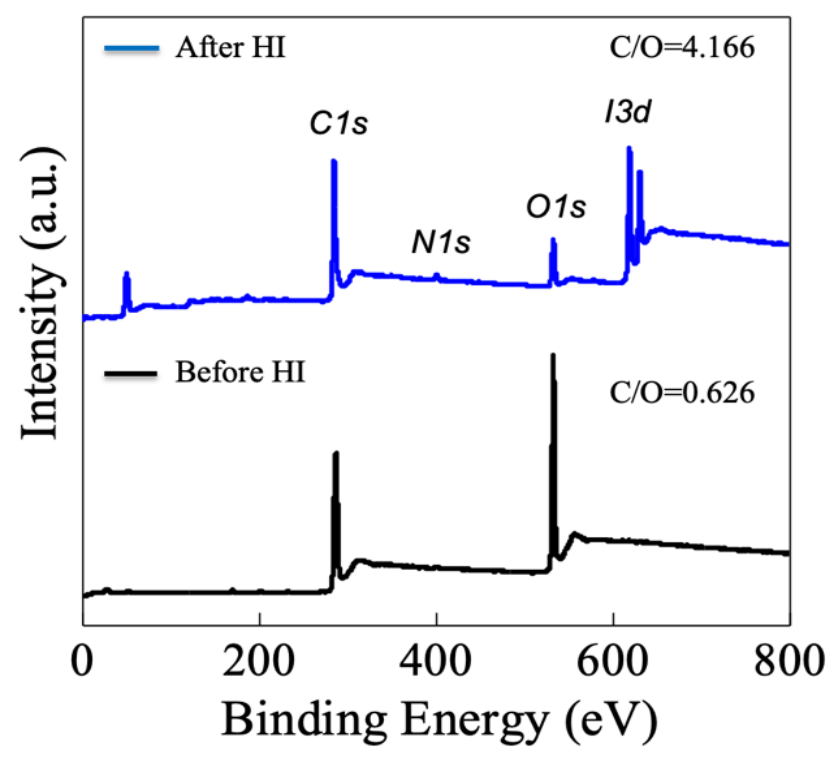

Figure S5. X-ray photoelectron spectroscopy (XPS) showed a change in diffraction peaks $(\mathrm{C} 1 \mathrm{~s}$, $O 1 s$, and $13 d$ ) of ASMGO before and after HI reduction in the binding-energy range from 0 to $800 \mathrm{eV}$. 


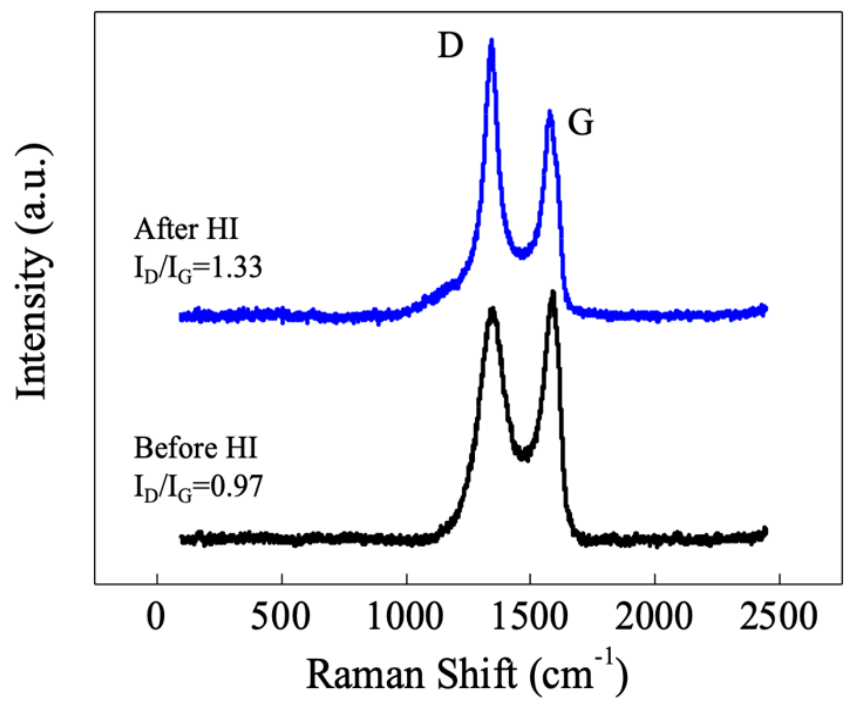

Figure S6. The $I_{D} / I_{G}$ ratio was calculated in the range from 0 to $2500 \mathrm{~cm}^{-1}$ in the Raman spectra; a change in the surface morphology and in the microstructure of ASMGO before and after HI reduction. 


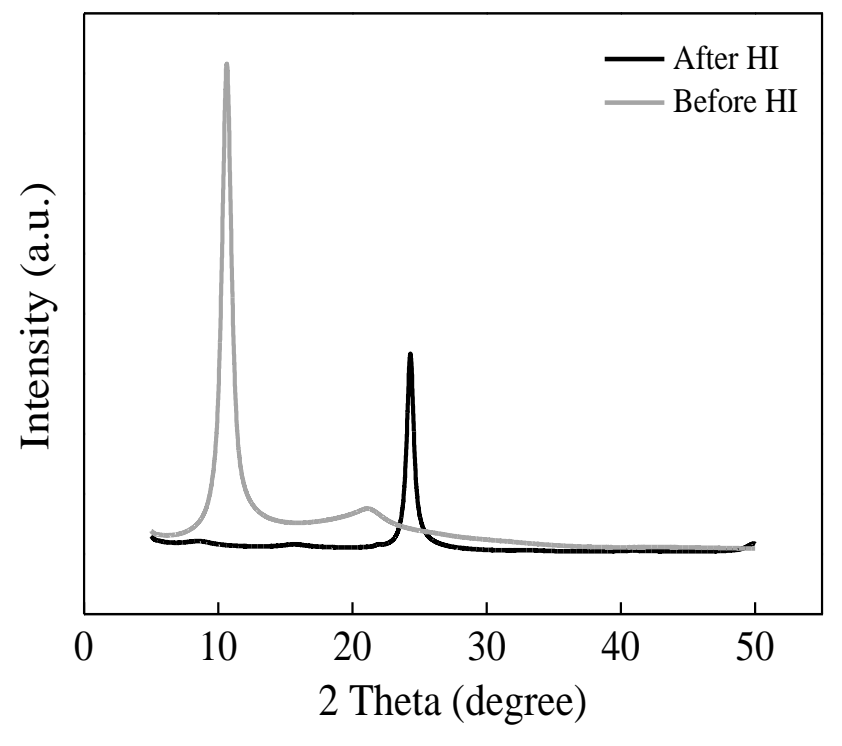

Figure S7. comparison of the XRD pattern of ASMGO before and after the HI reduction at $0-$ 50 of 2 Theta (degree) 


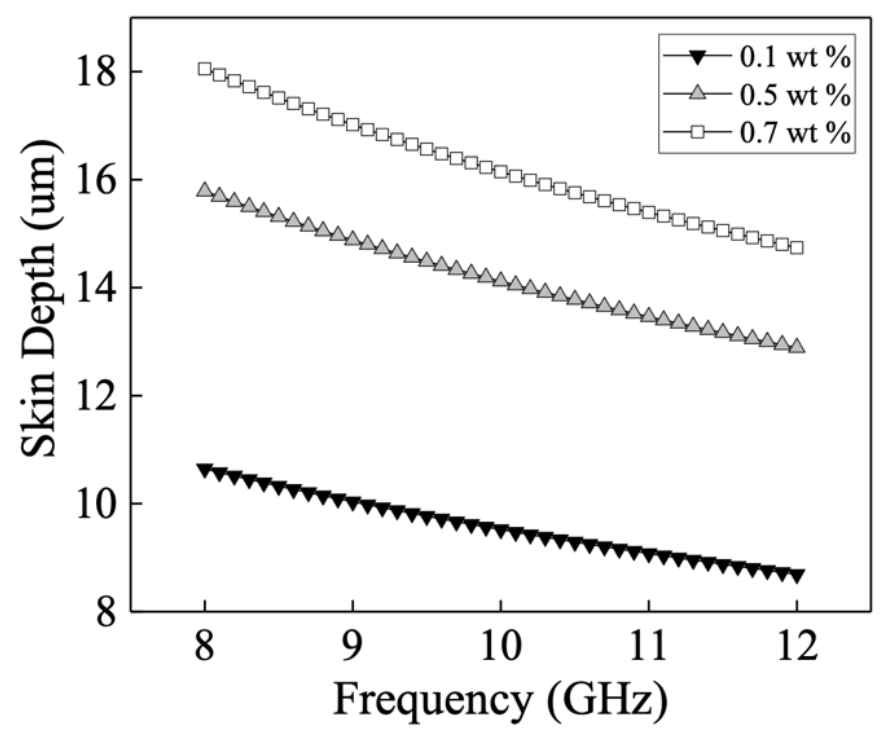

Figure S8. Skin depths of ASMGO (r-LCGO: 0.1, 0.5, and 0.7 wt\%) in the X-band frequency range (8.2-12.4 GHz) based on Equation (1).

These skin depths decrease as the frequency increases as well as they are relatively higher than normal metals (eg., gold, silver, and aluminum). As the concentration (wt $\%$ ) of $r-$ LCGO increases, value of the skin depth is high and its reduction rate decreases in the X-band. That is, the higher the conductivity, the lower the rate of decrease while the frequency increases. 


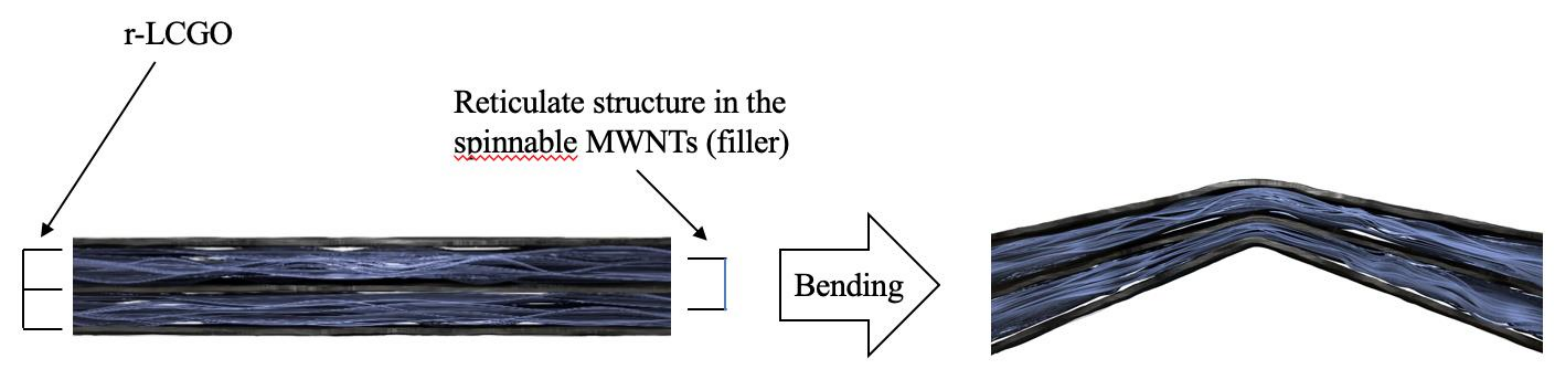

Figure S9. Cross-sectional views showing the role of the reticulated structure in the spinnable MWNTs when ASMGO was bent. The MWNTs served as a cushioning material between the r-LCGO layers. 
a)

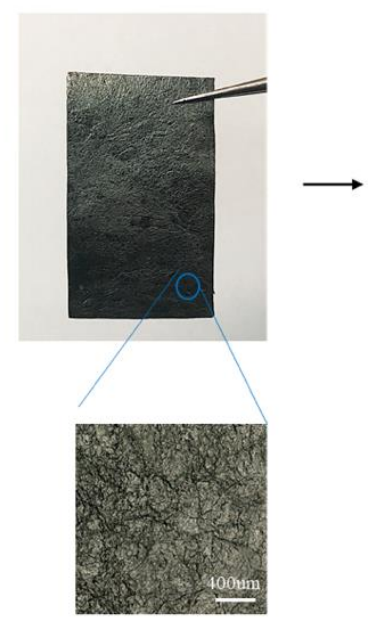

b)

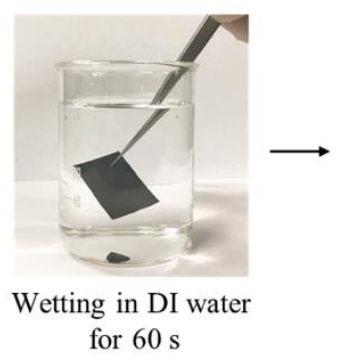

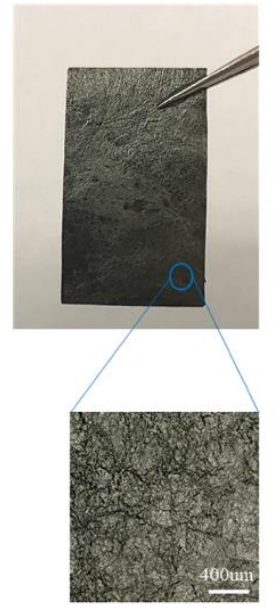

Water Proof

Figure S10. These figures demonstrate a) a hydrophobicity of ASMGO subjected to the HI reduction, through a wetting experiment in distilled water (DI) water for 60s, and b) hydrophobicity of carbon paper crane floated on water in a beaker.

The purpose of this additional experiment was to clearly confirmed if there were an organization of molecules or nonpolar groups to exclude water in an aqueous environment. As the above results, hydrophobicity in ASMGO make the carbon paper crane float on water, which showed excellent durability and sustainability even though a type of film ASMGO was in the water. Therefore, the non-wettability of ASMOG could apply to self-cleaning textiles, a surface of aerospace, and non-rust of bottom of a ship. 


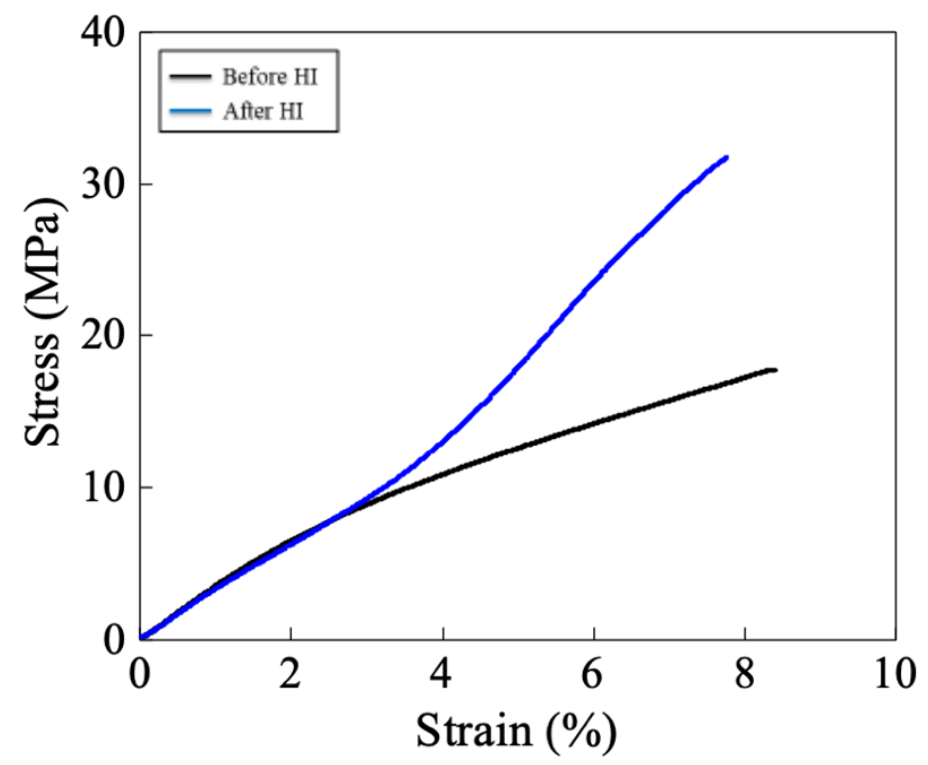

Figure S11. Strain-stress curves and toughness of ASMGO consisting of OSPM and LCGO before HI reduction (black line) and after HI reduction (blue line). The tensile speed was 1 $\mathrm{mm} / \mathrm{min}$ (EZ-SX, SHIMADZU. Co., Japan).

Basically, the well-aligned MWNT sheets layers between r-LCGO films increased the mechanical strength, but also orthogonally constructed MWNT sheets, which called a laminate structure, were advantageous for tension force from multi-direction. Additionally, by carrying out HI reduction, the functional groups in the graphene oxide were disappeared and then the LCGO film be much dense. Therefore, its mechanical strength and volumetric toughness were reinforced $80.1 \%$ and $27.6 \%$, respectively. In particular, the increased volumetric toughness (blue area-black area), which was its ability to absorb energy in the plastic range of the material, could be calculated by the area of the stress-strain curves as shown in Figure S9. 
a)

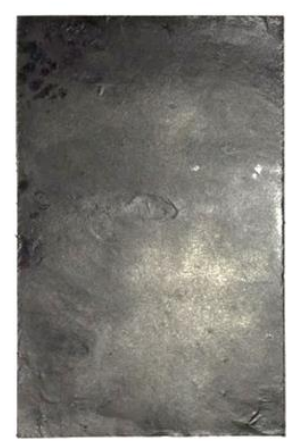

$11 \times 7 \mathrm{~cm}$

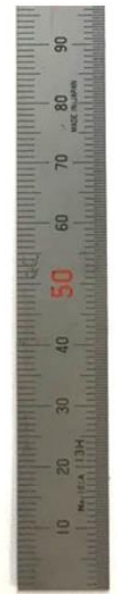

b)

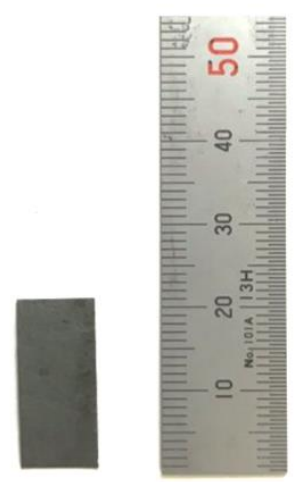

$2 \times 1 \mathrm{~cm}$

Figure S12. The ASMGO film for a) $11 \times 7 \mathrm{~cm}$, and b) $2 \times 1 \mathrm{~cm}$. 


\begin{tabular}{|c|c|c|c|c|c|c|c|c|}
\hline \multicolumn{2}{|c|}{ Type } & Filler & $\begin{array}{l}\text { Filler } \\
\text { [wt \%] }\end{array}$ & $\begin{array}{l}\text { Thickness } \\
{[\mathrm{cm}]}\end{array}$ & $\begin{array}{l}\text { EMI SE } \\
\text { [dB] }\end{array}$ & $\begin{array}{l}\text { *Specific } \\
\text { EMI SE (SSE) } \\
{\left[\mathrm{dB}^{3} / \mathrm{g}\right]}\end{array}$ & $\begin{array}{l}* * \mathrm{SSE} / \mathrm{t} \\
{\left[\mathrm{dB} \mathrm{cm}^{2} / \mathrm{g}\right]}\end{array}$ & Ref \\
\hline \multirow{2}{*}{\multicolumn{2}{|c|}{ Carbon }} & $\begin{array}{l}\text { CDG/PM } \\
\text { MA foam }\end{array}$ & 5 & 0.24 & 19 & $17-25$ & 70.8 & $(15)$ \\
\hline & & $\begin{array}{l}\mathrm{CNT} / \mathrm{PS} \\
\text { foam }\end{array}$ & 7 & 0.12 & 19 & 33 & 275 & (39) \\
\hline \multirow{4}{*}{ 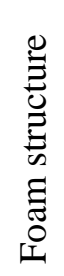 } & \multirow{4}{*}{ 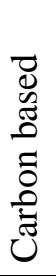 } & $\mathrm{rGO}$ & 10 & 0.08 & 21 & 937 & 11712 & (16) \\
\hline & & Carbon & - & 0.2 & 51.2 & 341 & 1705 & $(40)$ \\
\hline & & MWCNT & 76.2 & 0.1 & 21.1 & 541 & 5410 & $(7)$ \\
\hline & & $\mathrm{rGO}$ & 30 & 0.20 & 29 & 64.4 & 257.6 & $(10)$ \\
\hline \multirow{6}{*}{ 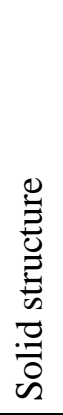 } & \multirow{6}{*}{ 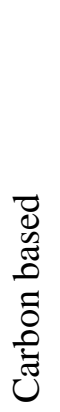 } & rGO & 7 & 0.25 & 45.1 & 173 & 692 & $(4)$ \\
\hline & & $\mathrm{rGO} / \mathrm{Fe}_{3} \mathrm{O}_{4}$ & Bulk & 0.03 & 24 & 31 & 1033 & $(30)$ \\
\hline & & $\mathrm{rGO}$ & 25 & 0.08 & 70 & 67.3 & 841 & $(38)$ \\
\hline & & MWNT & 15 & 0.11 & 50 & 47.6 & 432.7 & (14) \\
\hline & & $\mathrm{CB}$ & 37.5 & 0.2 & 18 & 30.3 & 15.1 & (36) \\
\hline & & CB & 15 & 0.11 & 20 & 20.9 & 190 & (14) \\
\hline \multirow{5}{*}{\multicolumn{2}{|c|}{$\begin{array}{l}\text { This } \\
\text { works }\end{array}$}} & \multirow{5}{*}{$\begin{array}{l}\text { r- } \\
\text { LCGO/S- } \\
\text { MWNTs }\end{array}$} & 0.1 & 0.0053 & 38.2 & 116.4 & 21953.5 & \\
\hline & & & 0.2 & 0.0064 & 40.1 & 90.8 & 14185.6 & \\
\hline & & & 0.3 & 0.0069 & 54.8 & 86.2 & 12488.3 & \\
\hline & & & 0.5 & 0.0076 & 66.1 & 50.2 & 6602.0 & \\
\hline & & & 0.7 & 0.0144 & 70.8 & 32.5 & 2259.1 & \\
\hline
\end{tabular}

Table S1. Comparison of the SSE and SSE/t in our work with those reported in other existing researches

The above Table S1 shows comparison our work with SSE and SSE/t of other existing researches. The SSE is defined as attenuation $(\mathrm{dB})$ per unit density of material, which mean that EMI SE of the material is divided by its density $\left(\mathrm{g} / \mathrm{cm}^{3}\right)$, mathematically. This value shows how many the amount of mass, related to EMI SE, contained per unit volume. Generally, a value of foam type of EMI SE materials is relatively high because it is thick and light. To obtain the SSE, we use the following equation; 
$* \mathrm{SSE}=\mathrm{EMI} \mathrm{SE} /$ density $=\mathrm{dB} \mathrm{cm}^{3} \mathrm{~g}^{-1}$

However, SSE has a critical limitation because it does not account for a thickness information. For example, it is very hard for a thick material to actually use for an application device even though it has high EMI SE. Therefore, it is necessary to recognize and understand SSE and SSE/t in order accurately to understanding EMI SE performance of a material, simultaneously. Generally, the larger this value, the greater the EMI SE value in a given thickness. To obtain $\mathrm{SSE} / \mathrm{t}$, we use the following equation;

$* * \mathrm{SSE} / \mathrm{t}=\mathrm{SSE} / \mathrm{t}($ thickness $)=\mathrm{dB} \mathrm{cm}^{2} \mathrm{~g}^{-1}$ 